\title{
Factores que influyen en el rendimiento académico de los estudiantes de la Facultad de Ciencias Matemáticas de la Universidad Nacional Mayor de San Marcos
}

\author{
Factors that influence in the academic performance of \\ the students of the Faculty of Mathematical sciences of \\ the Universidad Nacional Mayor de San Marcos
}

\author{
Carlos César Trucíos Maza ${ }^{a}$, María Estela Ponce Aruneri ${ }^{b}$
}

\begin{abstract}
RESUMEN
El rendimiento académico de los estudiantes de la Facultad de Ciencias Matemáticas de la Universidad Nacional Mayor de San Marcos, es una de las principales preocupaciones de los docentes y estudiantes de esta Facultad; nuestra investigación establecerá cuáles son los factores que influyen en su rendimiento. Seleccionamos una muestra aleatoria estratificada con selección sistemática de estudiantes, a los que se les administró un cuestionario que diseñamos para esta investigación, a los datos obtenidos se les aplicó Componentes Principales Categóricos (CATPCA) para reducir variables y Regresión Logística para encontrar los principales factores que influyen en el rendimiento académico de los estudiantes matriculados en el semestre académico 2008-I.
\end{abstract}

Palabras Clave: Componentes Principales Categóricos (CATPCA), Factores, Regresión Logística, Rendimiento Académico.

\begin{abstract}
The academic performance of students of the Faculty of Mathematical Sciences at the Universidad Nacional Mayor de San Marcos is one of the major concerns of teachers and students of the Faculty, our research will establish what factors influence their performance. We selected a stratified random sample with systematic selection of students, who were administered a questionnaire designed for this research, to data obtained were applied Categorical Principal Components (CATPCA) to reduce variables and logistic regression to find the main factors influencing in the academic performance of students enrolled in the semester, 2008-I.
\end{abstract}

Keywords: Categorical Principal Components (CATPCA), Factors, Logistic Regression, Academic Performance.

\section{INTRODUCCIÓN}

En la Facultad de Ciencias Matemáticas el porcentaje de estudiantes con promedio ponderado desaprobatorio fue el siguiente: $37.6 \%$ en el 2004[1], se incremento a $39.8 \%$ en el 2006 [2] y fue de $39.6 \%$ en el 2007 [3].
En el año 2007 [3] las Escuelas Académico Profesionales de la Facultad de Ciencias Matemáticas: Matemática (46.51\%), Estadística (31.75\%), Investigación Operativa (30.45\%) y Computación Científica (56.52\%); conjuntamente con las Escuelas de Química $(31.20 \%)$, Física $(36.14 \%)$ e Ingeniería

\footnotetext{
a Estudiante de la EAP de Estadística, Facultad de Ciencias Matemáticas, UNMSM, Lima - Perú

b Mg. en Estadística. Instituto de Investigación de la Facultad de Ciencias Matemáticas, UNMSM, Lima - Perú.
} 
Eléctrica (34.98\%) fueron las que tuvieron el mayor porcentaje de desaprobados en la UNMSM .

Investigaciones realizadas en el extranjero como en Madrid [4], y Maracaibo [5], estudiaron los factores que afectan al rendimiento académico, pero estas investigaciones no utilizaron métodos multivariados, sólo abordaron el problema de forma univariada o cualitativa, excepto en la investigación realizada en Madrid [6] en el 2000 que utiliza regresión logística, pero esta investigación solo considero a estudiantes del primer año. En el Perú no hemos encontrado, por lo menos hasta la fecha, investigaciones que aborden el problema del rendimiento académico con una metodología estadística que considere el análisis conjunto de las variables.

El rendimiento académico depende de muchos factores, el objetivo del presente trabajo es determinar cuáles son los principales factores que influyen en el rendimiento académico de los estudiantes de la Facultad de Ciencias Matemáticas de la UNMSM matriculados en el semestre académico 2008-I.

Los objetivos específicos considerados son: identificar cuáles son los factores que disminuyen la probabilidad de que un estudiante muestre un rendimiento académico satisfactorio, formular un modelo que permita predecir si un estudiante obtendrá un rendimiento académico satisfactorio; verificar si existen diferencias en el rendimiento académico por género, por Escuela Académico Profesional y entre la proporción de estudiantes aprobados y desaprobados.

\section{MATERIAL Y MÉTODOS}

Se elaboró el diseño muestral adecuado para la población bajo estudio y se utilizaron los siguientes métodos multivariados: Análisis de Componentes Principales Categóricos (CATPCA) [7] para reducir la dimensión de las variables y Regresión Logística [8] para encontrar los principales factores que influyen en el rendimiento académico de los estudiantes matriculados en el semestre académico 2008-I.

\section{DISEÑO MUESTRAL}

El marco muestral utilizado fue la relación de estudiantes matriculados en el semestre académico 2008-I.

Con el objetivo de obtener una muestra representativa de los estudiantes de la Facultad de Ciencias Matemáticas de la Universidad Nacional Mayor de San Marcos, se diseño una muestra probabilística estratificada con selección sistemática [9] dentro de cada estrato, se consideró un nivel de confiabilidad del $5 \%$ y un error de muestreo de 0.08 .

El tamaño de muestra se obtuvo de:

$$
n \geq \frac{\left(\sum_{h=1}^{L} W_{h} \frac{N_{h} P_{h} Q_{h}}{N_{h}-1}\right)^{2}}{\frac{e^{2}}{Z_{\alpha}^{2}}+\frac{1}{N} \sum_{h=1}^{L} W_{h}\left(\frac{N_{h} P_{h} Q_{h}}{N_{h}-1}\right)^{2}}=\frac{0.2305}{0.0017}=135.58
$$

La muestra fue de 136 estudiantes. La distribución de la muestra en cada uno de los estratos se realizó por afijación proporcional al tamaño.

\section{DISEÑO DEL INSTRUMENTO PARA RECOLECTAR LOS DATOS}

Con el objetivo de recoger los datos necesarios para realizar el análisis se elaboró un cuestionario para la presente investigación, el que contenía mayoritariamente preguntas cerradas; para la elaboración del cuestionario se tomó como referencia algunas de las preguntas consideradas en los trabajos [4],[5] y [6]; además de preguntas sugeridas por algunos docentes y estudiantes, que consideramos relevantes.

\section{ANÁLISIS DE COMPONENTES PRINCIPALES CATEGÓRICOS}

Análisis de componentes principales categóricos (CATPCA) [10], es una técnica multivariada que permite reducir un número grande de variables a un grupo pequeño de nuevas variables incorrelacionadas; está incluido en la categoría de los métodos de escalamiento óptimo; la diferencia con respecto al análisis de componentes principales tradicional radica en que el CATPCA se utiliza cuando las relaciones entre variables 
cuantitativas son no lineales y/o cuando las variables bajo estudio son categóricas.

El escalamiento óptimo asigna cuantificaciones numéricas a las categorías de cada variable. La cuantificación óptima de cada variable escalada se obtiene con el método de mínimos cuadrados alternantes; este método es iterativo.

\section{MODELO DE REGRESIÓN LOGÍSTICA}

La Regresión Logística [11] investiga y cuantifica la relación existente entre un conjunto de variables explicativas con una variable de respuesta binaria; determina las variables que influyen sobre la ocurrencia o no ocurrencia de un fenómeno; predice si un fenómeno va a ocurrir o no, y a la vez permite clasificar a los individuos en uno de los grupos definidos, en base a su probabilidad estimada.

El modelo de regresión logística:

$$
P\left(y_{i}=1 / \vec{x}_{i}\right)=\frac{e^{\beta_{0}+\beta_{1} x_{i 1}+\ldots \ldots \ldots .+\beta_{k} x_{i k}}}{1+e^{\beta_{0}+\beta_{1} x_{1 i}+\ldots \ldots \ldots .+\beta_{k} x_{i k}}}
$$

indica la probabilidad de que el i-ésimo estudiante de la Facultad de Ciencias Matemáticas (FCM) de la UNMSM, que se matriculo en el semestre académico 2008-I tenga un rendimiento académico satisfactorio.

Para que las variables incluidas en el modelo sean relevantes, se realizó previamente una selección de las mismas, utilizando el Método Backward-Wald [8].

Se utiliza el método de Newton Rapson[8] para calcular los estimadores de máxima verosimilitud del modelo propuesto, la función a maximizar es:

$$
L\left(y_{1}, \ldots, y_{n}, \beta\right)=\prod_{i=1}^{n} P\left(Y=y_{i}\right)=\prod_{i=1}^{n} \pi_{i}^{y_{i}}\left(1-\pi_{i}\right)^{1-y_{i}}
$$

Evaluamos la significancia del modelo, parámetros y bondad de ajuste; utilizando la prueba de razón de verosimilitud, la estadística de Wald y la prueba de Hosmer y Lemeshow, respectivamente[8].

Para detectar la presencia de observaciones atípicas y/o influyentes utilizamos las siguientes estadísticas [8]:

i) Distancia de Cook, detecta observaciones influyentes sobre las estimaciones de los parámetros del modelo. ii) Estadística delta Chi-cuadrado de Pearson y la estadística delta Chi-cuadrado de la desvianza, identifican observaciones que influyen en el valor ajustado del modelo propuesto, es decir que tan pobremente el modelo ajusta a la i-ésima observación.

Para identificar los factores que influyen en que un estudiante de la FCM-UNMSM matriculado en el semestre académico 2008-I muestre rendimiento académico satisfactorio, analizamos la significancia de las variables consideradas en el modelo y los valores de los odds ratio [11]; para evaluar la capacidad predictiva del modelo interpretamos los resultados de la tabla de clasificación y el índice gamma.

\section{PRUEBAS DE HIPÓTESIS}

La prueba de hipótesis para diferencia de proporciones [12], nos permite contrastas si existe o no diferencia entre las proporciones de una determinada característica para dos poblaciones, el estadístico de prueba esta dado por:

$$
Z=\frac{\left(p_{A}-p_{B}\right)-\left(p_{A}-p_{B}\right)}{S}
$$

Donde:

$$
S=\sqrt{P(1-P)\left(\frac{1}{n_{A}}+\frac{1}{n_{B}}\right)} \quad P=\frac{n_{A} \times p_{A}+n_{B} \times p_{B}}{n_{A}+n_{B}}
$$

\section{RESULTADOS Y DISCUSIÓN}

\section{MUESTRA}

El tamaño de muestra obtenido bajo el diseño muestral propuesto en (1) fue de 136, sólo se logró aplicar el cuestionario a 121 alumnos, debido a imprevistos ocurridos en la UNMSM, quedando la distribución de la muestra obtenida:

Tabla 1: Distribución de la muestra según Género y Escuela Académico Profesional

\begin{tabular}{lccc}
\hline \multirow{2}{*}{ EAP } & \multicolumn{2}{c}{ GËNERO } & Total \\
\cline { 2 - 3 } & Masculino & Femenino & \\
\hline Matemática & 26 & 7 & 33 \\
Estadística & 17 & 17 & 34 \\
I. Operativa & 17 & 23 & 40 \\
C. Científica & 10 & 4 & 14 \\
\hline Total & 70 & 51 & 121 \\
\hline \multicolumn{2}{c}{ Fuente: Trucios C.- Ponce E. }
\end{tabular}




\section{CUESTIONARIO AUTOADMINISTRADO, DETALLES DE LA OBTENCIÓN DE LOS DATOS}

La recolección de los datos se realizó entre la tercera semana de setiembre y octubre, se contó con la colaboración de cuatro estudiantes de la Facultad, a los que se les proporcionó los nombres de las personas, horarios y lugares donde se les podía ubicar, el cuestionario fue entregado y devuelto el mismo día; debido a la poca disponibilidad de tiempo de algunos estudiantes para completar el cuestionario (se rehusaron a colaborar con el estudio), se reemplazo a los estudiantes faltantes (aproximadamente $30 \%$ ) por estudiantes con las mismas características (género, Escuela Académico Profesional y año de ingreso).

Se elaboró un cuestionario con 51 preguntas y con 5 secciones:

I. Datos Generales

II. Antecedentes Académicos

III. Nivel Socio Económico

IV. Actitudes hacia el estudio

V. Servicios brindados por la biblioteca

\section{ANÁLISIS DE COMPONENTES PRINCIPALES CATEGÓRICOS}

Se realizó el CATPCA en cuatro conjuntos de variables, con el objetivo de poder reducir el número de variables a utilizar en el análisis de regresión logística. Finalmente el número de conjuntos con mayor variabilidad fueron tres, utilizamos la primera componente de cada uno de los tres conjuntos debido a que es la que concentra la mayor variabilidad de las variables que la componen.

Tabla 2: Saturaciones de la componente para el primer conjunto de variables: Nivel Socioeconómico del estudiante

\begin{tabular}{|l|c|}
\hline & \\
\cline { 2 - 2 } & \\
\hline Nivel de estudios más alto alcanzado por los padres & -.384 \\
Ocupación del padre o madre & .251 \\
Homo Microondas & .648 \\
Servicio de cable & .636 \\
Equipo de sonido & .552 \\
Televisor a colores & .324 \\
Camara digital & .728 \\
Servicio de internet & .610 \\
Lustradora & .621 \\
DVD & .562 \\
Lavadora & .619 \\
Aspiradora & .588 \\
Impresora & .666 \\
Filmadora & .591 \\
Computadora & .572 \\
\hline
\end{tabular}

La nueva componente que muestra la Tabla 2 , está estrechamente correlacionada con la tenencia de ciertos artículos electrodomésticos y servicios del hogar (valor superior a 0.5), esta nueva componente nos indica que a mayor valor de la misma se presenta una menor tenencia de artículos en el hogar, esta componente es un indicador del nivel socio económico del estudiante.

Tabla 3: Saturaciones de la componente para el segundo conjunto de variables: Servicios utilizados y brindados por la biblioteca

\begin{tabular}{|l|l|}
\hline \multirow{2}{*}{} & \\
\cline { 2 - 2 } & \\
\hline Consultar libros de la biblioteca & .810 \\
Realizar consultas por intemet & .764 \\
Estudiar con sus libros y/o apuntes & .800 \\
Consultar tesis y/o monografias & .618 \\
\hline
\end{tabular}

La componente obtenida en la Tabla 3, segundo conjunto de variables, esta correlacionada con los servicios brindados por la biblioteca de la Facultad; una mayor puntuación en esta componente indica un menor uso de los servicios de la biblioteca, esta componente es un indicador del uso de los servicios brindados por la biblioteca.

La tercera y última componente se obtiene del tercer conjunto de variables, cuyos resultados se muestran en la Tabla 4, nos indica que a mayor valor de esta componente, el estudiante es responsable con respecto a su rendimiento académico, sin embargo presenta algunas dificultades psicológicas y físicas que pueden estar afectando su rendimiento.

Tabla 4: Saturaciones de la componente para el tercer conjunto de variables: Actitudes y características psicológicas y físicas

\begin{tabular}{|l|c|}
\hline \multicolumn{1}{l|}{} & \\
\cline { 2 - 2 } & \\
\hline Percepción de capacidad para la carrera con respecto a la de sus & -.108 \\
Compañeros & \\
¿Entiende las orientaciones o guías de trabajo que proporciona el & .724 \\
profesor? & .596 \\
Interés con respecto a sus calificaciones & .555 \\
¿Desarrolla usted sus tareas, ejercicios o trabajos? & .369 \\
Actitud frente a los exámenes & .468 \\
Actitud con respecto al cumplimiento de sus tareas y/o trabajos en el & .522 \\
tiempo previsto & .313 \\
¿Le duele la cabeza cuando lee o estudia? & .520 \\
¿Se distrae con frecuencia en clases? & .365 \\
¿Cuándo se pone a estudiar le da sueño? & .152 \\
¿Cuándo lee vienen a su mente ideas ajenas al texto que esta leyendo? & .521 \\
¿Memoriza las demostraciones y ejercicios resueltos en clase sin &. .687 \\
entenderlos? & .570 \\
¿Se cansa de estudiar después de media hora? & -.373 \\
¿Elude estudiar los cursos más difíciles? & .371 \\
¿Se siente cansado constantemente? & \\
¿Sus profesores resuelven sus consultas o dudas? & \\
Apreciación del dictado de clase se sus profesores &
\end{tabular}




\section{MODELO DE REGRESIÓN LOGÍSTICA}

\section{Selección de variables y estimación de los parámetros del modelo}

El método de selección de variables BackwardWald [8], proporcionó el conjunto de variables relevantes para construir el modelo de regresión; las variables seleccionadas fueron:

a) Del grupo antecedentes académicos: tipo de colegio donde realizó sus estudios secundarios, institución donde se preparó para ingresar a la universidad,

b) Del grupo socioeconómico: lugar donde toma sus alimentos, apoyo económico familiar.

c) Servicios brindados por la biblioteca.

Estas variables son las que mayor información aportan para estimar la probabilidad de que un estudiante de la FCM-UNMSM matriculado en el semestre académico 2008-I muestre un rendimiento académico satisfactorio. Así el modelo propuesto para conocer los factores que influyen en el rendimiento académico y que permiten estimar la probabilidad de que un estudiante obtenga un rendimiento académico satisfactorio es de la forma:

$P\left(y_{i}=1 / \vec{x}_{i}\right)=\frac{e^{\beta_{1} x_{i 1}+\beta_{2} x_{i 2}+\beta_{3} x_{i 3}+\beta_{4} x_{i 4}+\beta_{5} x_{i 5}}}{1+e^{\beta_{1} x_{i 1}+\beta_{2} x_{i 2}+\beta_{3} x_{i 3}+\beta_{4} x_{i 4}+\beta_{5} x_{i 5}}}$

y donde los valores de los $\beta_{i}$ están definidos según la siguiente tabla:

Tabla 5: Estimación y Evaluación de la significancia de los parámetros del modelo

\begin{tabular}{|c|c|c|c|c|}
\hline & B & $\begin{array}{l}\text { Estadistico } \\
\text { de Wald }\end{array}$ & P-Valor & $\begin{array}{r}\text { Odd } \\
\text { Ratio }\end{array}$ \\
\hline TIPO DE COLEGIO (Privado) & -1.509 & 5.779 & .016 & .22 \\
\hline TIPO DE PREPARACIÓN & & 6.051 & .049 & \\
\hline CEPUSM & 1.588 & 3.867 & .049 & .048 \\
\hline Individual & 1.731 & 3.496 & .062 & .062 \\
\hline $\begin{array}{l}\text { LUGAR DONDE TOMA SUS } \\
\text { ALIMENTOS }\end{array}$ & & 4.912 & .086 & \\
\hline Restaurantes / Pensión & -.678 & 1.035 & .309 & .508 \\
\hline Comedor universitario & -1.500 & 4.802 & .028 & .223 \\
\hline APOYOECONÓMICO & & 21.217 & .000 & \\
\hline Parcial & 2.773 & 18.341 & .000 & 16.000 \\
\hline Total & 2.492 & 14.239 & .000 & 12.082 \\
\hline $\begin{array}{l}\text { USO DE LOS SERVICIOS } \\
\text { BRINDADOS POR LA BIBUOTECA }\end{array}$ & & 26.412 & .000 & \\
\hline Medio & 1.138 & 2.194 & .139 & 3.120 \\
\hline Poco & -2.820 & 18.728 & .000 & .060 \\
\hline
\end{tabular}

\section{2ํㅡㄹ Evaluación de la significancia del modelo}

Probaremos la siguiente hipótesis:

Ho $: \beta_{1}=\beta_{2}=\ldots \ldots \ldots \ldots \ldots . . . .=\beta_{9}=0$
Con el estadístico -2 Ln de la verosimilitud $=$ 88.13 y $\chi_{9}^{2}=2.7$

Para un nivel de significación del $5 \%$, se tiene que el modelo es significativo. Es decir al menos uno de los parámetros es diferente de cero y por lo tanto al menos uno de los factores o variables consideradas influye en el rendimiento académico de los estudiantes de la FCMUNMSM matriculados en el semestre académico 2008-I.

\section{Evaluación de la significancia individual de los parámetros del modelo.}

Considerando los resultados del estadístico de Wald, las hipótesis en este caso son:

$$
\begin{array}{ll}
\mathbf{H o :}: \beta_{\mathrm{i}}=0 & \mathrm{i}=1, \ldots \ldots, 9 \\
\mathbf{H}_{1}: \beta_{\mathrm{i}} \text { ' } 0 & \text { para al menos un « } \mathrm{i} \text { » }
\end{array}
$$

Si observamos los p-valores [12] de la Tabla 5; podemos concluir que todas las variables son significativas en al menos una de sus categorías, con un nivel de significación del $5 \%$

\section{4 ํvaluación de la bondad de ajuste del modelo.}

Se utiliza el test de Hosmer y Lemeshow [8], que se basa en la estadística Chi-cuadrado.

Las hipótesis a probar son:

Ho: No existe diferencia entre los valores observados y los estimados a partir del modelo de regresión logística.

$\mathbf{H}_{1}$ : Existe diferencia entre los valores observados y los estimados a partir del modelo de regresión logística.

Tabla 6: Test Hosmer y Lemeshow

\begin{tabular}{ccc}
\hline Estadística Chi-cuadrado & $\begin{array}{c}\text { Grados de } \\
\text { libertad }\end{array}$ & p-valor \\
\hline 7.57016 & 8 & 0,77 \\
\hline
\end{tabular}

Los resultados de la Tabla 6, indica que el modelo explica adecuadamente el comportamiento de las variables que muestra la Tabla 5. 


\section{Detección de observaciones atípicas y/ 0 influyentes}

Utilizaremos métodos gráficos para mostrar el comportamiento de las estadísticas [8] que permitirán detectar las observaciones que influyen en las estimaciones del modelo propuesto.

A partir del modelo estimado y de los valores de las variables, calculamos [8] la probabilidad estimada, los valores leverage, la distancia de Cook, la estadística delta chi-cuadrado y delta desvianza; y los DF BETAS para cada uno de los parámetros.

Tabla 7: Posibles observaciones influyentes

\begin{tabular}{lc}
\hline MÉTODO & OBSERVACIONES \\
\hline DFBETA1 & 110 \\
DFBETA2 & 34 \\
DFBETA3 & 33,57 \\
DFBETA4 & $33,19,58$ \\
DFBETA5 & $73,84,88$ \\
DFBETA6 & - \\
DFBETA7 & 95 \\
DFBETA8 & 19,99 \\
DFBETA9 & - \\
\hline
\end{tabular}

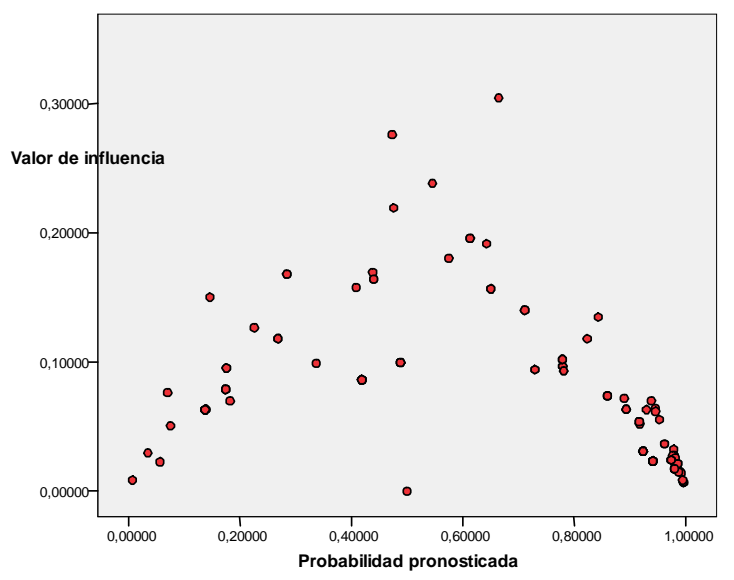

Gráfico 1. Probabilidad estimada y Valores Leverage

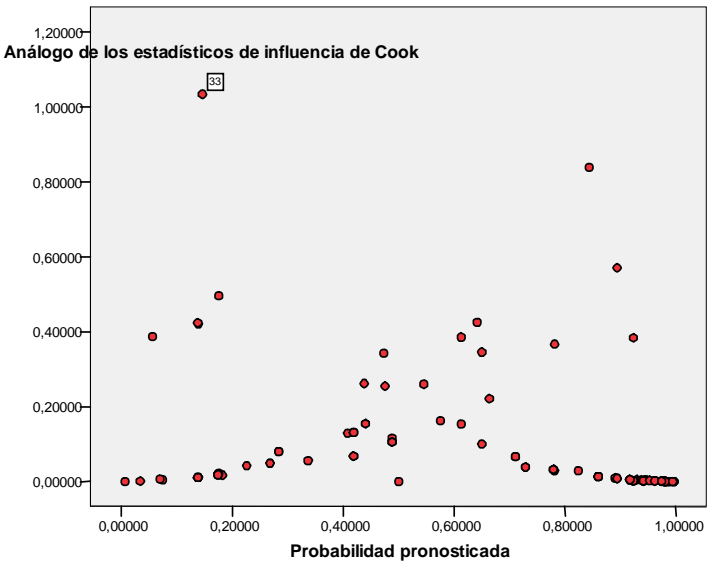

Gráfico 2. Probabilidad estimada y Distancia de Cook

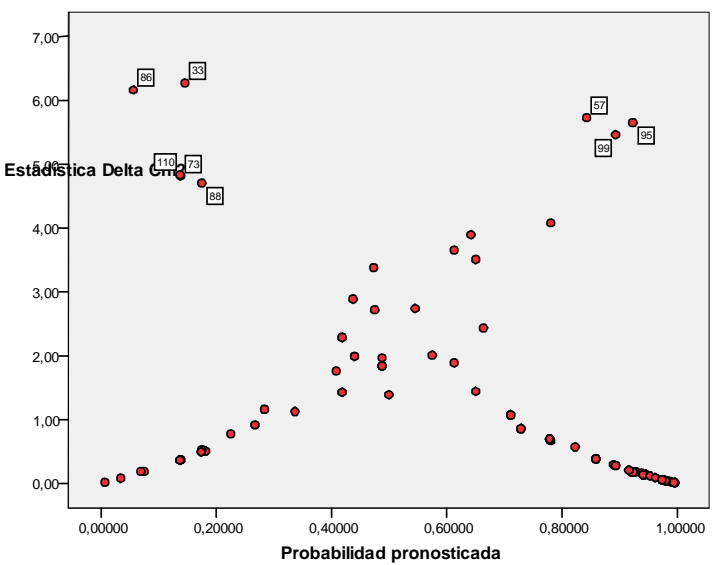

Gráfico 3. Probabilidad estimada y estadístico Delta

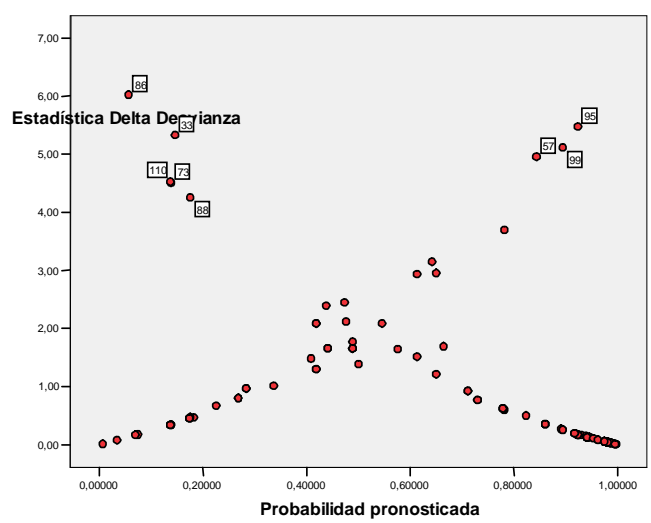

Gráfico 4: Probabilidad estimada y estadística Delta Desvianza

Los gráficos 1, 2, 3 y 4, y la tabla 7, muestran las posibles observaciones influyentes [8], se realizó un análisis posterior, retirando dichas observaciones del conjunto de datos y 
observamos que los resultados de la regresión logística no presentan cambios significativos.

\section{Evaluación de la capacidad predictiva del modelo}

i) Tabla de Clasificación

Tabla 8: Capacidad Predictiva del modelo

\begin{tabular}{lccc}
\hline & \multicolumn{2}{c}{ PRONOSTICADO } & \\
\cline { 2 - 3 } OBSERVADO & Desaprobado & Aprobado & TOTAL \\
& & & \\
\hline Desaprobado & 30 & 7 & $81.1 \%$ \\
Aprobado & 9 & 75 & $89.3 \%$ \\
TOTAL & & & $86.8 \%$ \\
\hline
\end{tabular}

Como el porcentaje de aciertos en la clasificación es del $86.8 \%$, podemos concluir que el modelo tiene una buena capacidad predicativa.

ii) Índice de eficacia predictiva

Tabla 9: Índice Gamma

\begin{tabular}{cc}
\hline Valor del Índice & Aprox. Sig. \\
\hline 0,946 & 0,000 \\
\hline
\end{tabular}

El valor del Índice Gamma [11] es 0,946, lo que indica una muy buena capacidad predictiva del modelo propuesto.

\section{7을 Interpretación de los parámetros estimados del modelo}

Los factores: apoyo económico parcial, apoyo económico total y utilizar con cierta frecuencia los servicios brindados por la biblioteca, influyen para que un estudiante de la FCM de la UNMSM, tenga un rendimiento académico satisfactorio; mientras que el proceder de colegio privado, el haberse preparado en el CEPUSM , tomar los alimentos en el comedor universitario y utilizar con poca frecuencia los servicios brindados por la biblioteca son factores que disminuyen la probabilidad de que un estudiante de la FCM de la UNMSM obtenga un rendimiento académico satisfactorio.
Los estudiantes que toman sus alimentos en el comedor universitario y proceden de colegio privado muestran una disminución del $78 \%$ en la probabilidad de obtener rendimiento académico satisfactorio, en cada caso.

\section{PRUEBA DE HIPÓTESIS}

Se realizaron pruebas de hipótesis para verificar si existe diferencia en la proporción [12] de estudiantes con un rendimiento académico satisfactorio, según género y Escuela Académico Profesional. Las pruebas de hipótesis se realizaron con nivel de significancia del $5 \%$ y los resultados se presentan a continuación:

1- Prueba de hipótesis para la diferencia de proporciones de estudiantes con rendimiento académico satisfactorio según género.

Sean las hipótesis [12]:

$$
\begin{aligned}
& H_{0}: P_{H}=P_{M} \\
& H_{1}: P_{H} \neq P_{M}
\end{aligned}
$$

Donde:

$P_{H}=$ Proporción de varones con rendimiento académico satisactorio $P_{M}=$ Proporción de mujeres con rendimiento académico satisfactorio

La estadística para la prueba es:

$Z=0.9449562$

$Z_{\alpha / 2}=1.96$

Con un nivel de significancia del $5 \%$ no tenemos evidencia suficiente para rechazar la hipótesis nula, con lo que concluimos que la proporción de estudiantes con rendimiento académico satisfactoria es la misma para varones y mujeres.

2- Prueba de hipótesis para la diferencia de proporciones de estudiantes con rendimiento académico satisfactorio según escuela académico profesional.

\section{Sea:}

$P_{n x t}=$ Proporción de estudiantes de Matemática con rendimiento académico satisactorio $P_{e s}=$ Proporción de estudiantes de Estadística con rendimiento académico satisfactorio $P_{c c}=$ Proporción de estudiantes de Comp. Gientífica con rendimiento académico satisfactorio $P_{i, o}=$ Proporción de estudiantess de Inv. Operativa con rendimiento académico satisfactorio

La siguiente tabla muestra las hipótesis propuestas y los resultados: 
Tabla 10: Pruebas de hipótesis para diferencia de proporciones de estudiantes con rendimiento académico satisfactorio por Escuela Académico Profesional

\begin{tabular}{|c|c|c|}
\hline$H_{0}: P_{m a t}=P_{e s t}$ & $Z=1.10731 \quad Z_{\alpha / 2}=1.96$ & $\begin{array}{l}\text { No rechazamos } \\
\text { la hipótesis nula }\end{array}$ \\
\hline$H_{0}: P_{\text {mat }}=P_{i . o}$ & $Z=0.090826 \quad Z_{\alpha / 2}=1.96$ & $\begin{array}{l}\text { No rechazamos } \\
\text { la hipótesis nula }\end{array}$ \\
\hline$H_{0}: P_{m a t}=P_{c . c}$ & $Z=0.65329$ & $\begin{array}{l}\text { No rechazamos } \\
\text { la hipótesis nula }\end{array}$ \\
\hline$H_{0}: P_{e s t}=P_{i . o}$ & $Z=1.06290$ & $\begin{array}{l}\text { No rechazamos } \\
\text { la hipótesis nula }\end{array}$ \\
\hline$H_{0}: P_{e s t}=P_{c . c}$ & $Z=1.55312$ & $\begin{array}{l}\text { No rechazamos } \\
\text { la hipótesis nula }\end{array}$ \\
\hline$H_{0}: P_{c . c}=P_{i . o}$ & $Z_{\alpha / 2}=1.96$ & $\begin{array}{l}\text { No rechazamos } \\
\text { la hipótesis nula }\end{array}$ \\
\hline
\end{tabular}

Con un nivel de significancia del $5 \%$ no tenemos evidencia suficiente para rechazar las hipótesis nulas, con lo que concluimos que la proporción de estudiantes con rendimiento académico satisfactorio por escuela académico profesional es la misma.

3o Prueba de hipótesis para verificar que la proporción de estudiantes desaprobados es menor a la de los aprobados.

$H_{0}: P_{D}>P_{A}$

$H_{1}: P_{D}<P_{A}$

$Z=\frac{0.306-0.694}{0.09752}=-3.97886$

$\alpha=0.05$

$Z_{\alpha / 2}=-1.645$

Re chazamos la hipotesis nula

Con un nivel de significancia del $5 \%$, concluimos que la proporción de estudiantes desaprobados es menor a la proporción de aprobados.

\section{CONCLUSIONES}

$1^{\circ}$ En la Facultad de Ciencias Matemáticas el porcentaje de estudiantes con promedio ponderado desaprobatorio fue el siguiente: $37.6 \%$ en el 2004 [1], 39.8\% en el 2006 [2], $39.6 \%$ en el 2007 [3] y en base a los datos recolectados para esta investigación estimamos que fue del $30.58 \%$ para el 2008.

La mayoría de estudiantes que ingresan a las diversas Escuelas Académico
Profesionales de la Facultad de Ciencias Matemáticas de la Universidad Nacional Mayor de San Marcos, son los que obtuvieron lo más bajos puntajes [13] en los exámenes de admisión. Este es uno de los factores que si bien no se ha considerado como variable de estudio, influye directamente en el rendimiento académico del estudiante.

$2^{\circ}$ Los factores que disminuyen la probabilidad de que un estudiante de la Facultad de Ciencias Matemáticas de la UNMSM, matriculado en el semestre 2008-I, muestre un rendimiento académico satisfactorio son:

1. Estudios secundarios concluidos en Institución Educativa de Gestión Privada.

2. Tomar sus alimentos en el comedor Universitario.

3. Utilizar con poca frecuencia los servicios que brinda la Biblioteca.

Se ha observado que los estudiantes que proceden de Instituciones Educativas Estatales son los que más se esfuerzan por mejorar su rendimiento académico y concluir su carrera profesional.

Los estudiantes que asisten a tomar sus alimentos (desayuno, almuerzo y cena) al Comedor Universitario, utilizan la mayor parte de su tiempo en hacer colas, por lo que no les queda tiempo suficiente para estudiar sus asignaturas y utilizar los servicios de la biblioteca.

$3^{\circ}$ El contar con apoyo económico familiar ya sea este parcial o total, contribuye a que el estudiante la Facultad de Ciencias Matemáticas de la UNMSM, muestre un rendimiento académico satisfactorio.

$4^{\circ}$ No existe diferencia en el rendimiento académico de los estudiantes de la FCM de la UNMSM matriculados en el semestre académico 2008-I, por género y Escuela Académico Profesional.

$5^{\circ}$ El porcentaje de estudiantes desaprobados sigue siendo menor al porcentaje de aprobados, en la FCM de la UNMSM; pero no baja del $30 \%$. 


\section{RECOMENDACIONES}

Se recomienda para trabajos futuros relacionados con el rendimiento académico, incrementar el tamaño de muestra utilizado, de preferencia el tamaño de muestra debe ser superior a diez veces el número de variables utilizadas en el modelo mas uno; para facilitar el proceso de la recolección de datos se recomienda utilizar muestreo bietápico.

\section{AGRADECIMIENTOS}

- A los siguientes estudiantes, quienes desinteresadamente colaboraron en la recolección de los datos: Janeth Cachay Mamani, Evelyn Sánchez Laureano, Priscila Condo Fernández y Juan Carlos Yataco Casas.

- Al Vicerrectorado Académico de la Universidad Nacional Mayor de San Marcos por el incentivo económico brindado para realizar esta investigación, como parte del programa «Proyectos de Iniciación Científica 2007»

\section{REFERENCIAS BIBLIOGRÁFICAS}

[1]. SUM Boletín Informativo No 13, Universidad Nacional Mayor de San Marcos. 2005. Lima, Perú.

[2]. SUM Boletín Informativo No 21, Universidad Nacional Mayor de San Marcos. 2007. Lima, Perú.

[3]. SUM Boletín Informativo No 25, Universidad Nacional Mayor de San Marcos. 2008. Lima, Perú.

[4]. Jano Salgare Dolores, Ortiz Serrano Salvador. 2004. Determinación de los factores que afectan al rendimiento académico en la educación superior. Madrid, España.

[5]. Hernández de Rincón A. 2005. El rendimiento académico de las matemáticas en alumnos universitarios. Maracaibo, Venezuela.

[6]. García Jiménez Visitación, Alvarado Izquierdo Jesús. 2000. La predicción del rendimiento académico: regresión lineal versus regresión logística. Madrid, España.

[7]. Heiser, Willen J, Meulman, J. 2005.SPSS Categories 14.0. E.E.U.U.

[8]. Hosmer David W. y Lemeshow Sanley. 2000. Applied Logistic Regression. John Wiley \& Sons. New York.

[9]. Peréz López César. 2000. Técnicas de Muestreo Estadístico. Alfaomega. México D.F.

[10]. Moroco Joao. 2003. Análise Estatistica, com utilizaçao do SPSS. Silabo. Lisboa.

[11]. Cea D Ancona María Angeles. 2002. Análisis Multivariable: Teoría y Práctica en la Investigación Social. Síntesis. Madrid.

[12]. Casas Sánchez José María, Zamora Sanz Ana Isabel. 1997. Inferencia Estadística. Editorial Ramón Areces. España.

[13]. Área de Prueba de la Oficina Central de Admisión. Informes de los Procesos de Admisión de la Universidad Nacional Mayor de San Marcos. 2006, 2007 y 2008. Lima, Perú.

E-mail: $\quad \underline{\text { ctrucios@gmail.com }}$

mepaunmsm@yahoo.es 